1 Title: Subsidies mediate interactions between communities across space.

2 Authors: Isabelle Gounand ${ }^{\star 1,2}$, Eric Harvey ${ }^{1,2}$, Pravin Ganesanandamoorthy ${ }^{2}$, Florian

3 Altermatt $^{1,2}$

4

5 Type of Article: Research paper

$7 \quad{ }^{*}$ Corresponding author:

8 Isabelle Gounand: isabelle.gounand@eawag.ch; ORCID: 0000-0002-0675-3973

9

10 E-mails:

11 EH Eric.Harvey@eawag.ch; ORCID: 0000-0002-8601-7326

12 PG Pravin.Ganesanandamoorthy@eawag.ch;

13 FA florian.altermatt@eawag.ch; ORCID: 0000-0002-4831-6958

\title{
15 Affiliations
}

16 'Department of Evolutionary Biology and Environmental Studies, University of Zurich,

17 Winterthurerstrasse 190, CH-8057 Zürich, Switzerland.

$18{ }^{2}$ Eawag, Swiss Federal Institute of Aquatic Science and Technology, Department of

19 Aquatic Ecology, Überlandstrasse 133, CH-8600 Dübendorf, Switzerland.

This document is the accepted manuscript version of the following article:

Gounand, I., Harvey, E., Ganesanandamoorthy, P., \& A1termatt, F. (2017). Subsidies mediate interactions between communities across space. 0ikos, 126(7), 972-979. https://doi.org/10.1111/oik.03922 


\section{Abstract}

Most spatial ecology focuses on how species dispersal affects community

23 dynamics and coexistence. Ecosystems, however, are also commonly connected by

24 flows of resources. We experimentally tested how neighbouring communities indirectly

25 influence each other in absence of dispersal, via resource exchanges. Using two-patch

26 microcosm meta-ecosystems, we manipulated community composition and dynamics,

27 by varying separately species key functional traits (autotroph versus heterotroph species

28 and size of consumer species) and trophic structure of aquatic communities (species

29 growing alone, or in presence of competitors or predators). We then analysed the effects

30 of species functional traits and trophic structure on communities connected through

31 spatial subsidies in the absence of actual dispersal. Both functional traits and trophic

32 structure strongly affected dynamics across neighbouring communities. Heterotroph

33 communities connected to autotroph neighbours developed better than with heterotroph

34 neighbours, such that coexistence of competitors was determined by the functional traits

35 of the neighbouring community. Densities in autotroph communities were also strikingly

36 higher when receiving subsidies from heterotroph communities compared to their own

37 subsidies when grown in isolated ecosystems. In contrast, communities connected to

38 predator-dominated ecosystems collapsed, without any direct contact with the predators.

39 Our results demonstrate that because community composition and structure modify the

40 distribution of biomass within a community, they may also affect communities connected

41 through subsidies through quantitative and qualitative changes of detritus flows. This

42 stresses that ecosystem management should account for such interdependencies

43 mediated by spatial subsidies, given that local community alterations cascade across 
44 space onto other ecosystems even if species dispersal is completely absent.

45 


\section{Introduction}

Spatial flows between ecosystems are ubiquitous in nature. Research on spatial

48 ecology and meta-community dynamics demonstrated the fundamental role of dispersal

49 for species coexistence and biodiversity at local and regional scales (Mouquet and

50 Loreau 2002, Leibold et al. 2004, Holyoak et al. 2005, Seymour et al. 2015). In parallel,

51 ecosystem ecology and ecosystem-level studies have shown that spatial flows of

52 resources are also fundamental drivers of community dynamics (Polis et al. 1997, 2004,

53 Harvey et al. 2016). In natural ecosystems, many communities have a net heterotrophic

54 functioning and productivity relies on subsidies coming from neighbouring ecosystems.

55 For instance benthic marine- or freshwater-systems rely on detritus inputs sinking from

56 pelagic waters (Fitzgerald and Gardner 1993, Schindler and Scheuerell 2002), and at

57 aquatic-terrestrial boundaries, ecosystems experience strong bi-directional resource-

58 exchanges, with riverine vegetation subsidizing river or lake communities with dead-

59 organic matter (Hall et al. 2000, Cole and Caraco 2001, Richardson et al. 2010), while

60 riparian systems benefit from nitrogen-rich inputs of emergent aquatic insects (Baxter et

61 al. 2005, Gratton and Vander Zanden 2009, Dreyer et al. 2015), or fish carcasses

62 (Hocking and Reimchen 2009). The quantification of these resource spatial flows

63 (thereafter called subsidies), and the recognition of their importance for local community

64 dynamics by meta-community ecology, eventually led to a formal integration of

65 community and ecosystem perspectives on spatial flows within the meta-ecosystem

66 framework, accounting for both organism dispersal and resource exchanges between

67 ecosystems (Loreau et al. 2003, Massol et al. 2011, Massol et al. in review).

$68 \quad$ Previous studies on allochthonous subsidies generally investigated subsidy 
69 effects on recipient communities from a donor-controlled perspective, thus without

70 consideration of the reciprocal effects on both connected ecosystems (e.g. Cole et al.

71 2006). Reframed into a spatial perspective, however, subsidies could be considered as

72 a vector of indirect interactions between community dynamics, with donor community

73 dynamics modulating the quantity and quality of exported resources. Both composition

74 (species traits) and structure (trophic interactions) of communities control the quantity

75 and quality of detritus locally produced, and potentially exported to other ecosystems as

76 subsidies. On the one hand, the population dynamics and life cycles of the species

77 composing a community determine the amount of detritus produced locally (e.g. subsidy

78 pulses triggered by 17-year emergence cycles of cicadas reported by Menninger et al.

79 2008). On the other hand, species functional traits determine detritus quality via their

80 stoichiometry (Sitters et al. 2015). Indeed, key species functional traits such as

81 autotrophy translate into carbon-nutrient ratios biased toward high values due to their

82 carbon-fixing photosynthesis activities, compared to the content of heterotrophic species

83 (see Sterner and Elser 2002, Sterner 2009 for cross-taxon comparisons). Through a

84 direct effect on the stoichiometric balance and the degradability of building block

85 molecules, community composition modulates not only detritus composition, but also the

86 rate at which detritus is made available as a new resource for subsidized communities

87 (Enriquez et al. 1993, Scott and Binkley 1997, Allison 2012), such as plant litter

88 composition driving decomposition rates in forest and stream ecosystems worldwide

89 (Cornwell et al. 2008, García-Palacios et al. 2015).

90 Community structure and the strength of species interactions within a community

91 should also affect the quantity and quality of detritus by modulating the biomass 
92 distribution across trophic levels (Shurin et al. 2006). Empirical studies, for instance,

93 showed that in a wide range of ecosystems, changes in one trophic level induce trophic

94 cascades over an entire food web, which leads to drastic changes in biomass

95 distributions (Schmitz et al. 2000, Carpenter et al. 2001, Jackson et al. 2001, Frank et al.

96 2005). Beyond the traditional focus on species loss and local productivity change, the

97 spatial consequences for subsidy-connected ecosystems of these structural changes

98 and the subsequent modified detritus production have still to be investigated.

Overall the tight link between community composition and dynamics, and local

100 detritus production triggers an indirect connection between communities connected by

101 subsidy exchanges: the recipient community fed by subsidies becomes indirectly linked

102 to the donor community dynamics through the qualitative and quantitative characteristics

103 of the exported resources. Such subsidy linkages between ecosystems imply that local

104 community composition and structure may matter for the functioning of neighbouring

105 communities even in the absence of species' dispersal. This hypothesis has

106 fundamental implications for the functioning of connected ecosystems and for their

107 response to anthropogenic pressure, since it postulates the spatial spread of local

108 perturbations. Any event that would modify local community composition and dynamics,

109 such as biological invasions, is susceptible to pass on neighbouring community

110 dynamics through subsidy disruption even if species dispersal cannot occur (e.g. Baxter

111 et al. 2004).

$112 \quad$ Here we propose a test of such indirect "neighbour effects" with experimental

113 microcosm meta-ecosystems. We used aquatic microbial communities consisting of

114 bacteria feeding on organic resources, various autotroph and bacterivorous protists, and 
115 a top predator. The microcosms were mimicking a wide range of natural ecosystems,

116 from heterotrophic, such as in stream or benthic waters, to autotrophic, such as forests

117 or pelagic phytoplankton-based ecosystems, and from resource to predator dominated.

118 We built two-patch meta-ecosystems connecting ecosystems only by spatial subsidies

119 (spatial flows of detritus and inorganic resources, and no organisms dispersing). In one

120 ecosystem we manipulated species traits (autotroph versus heterotroph species and

121 consumer body size), while in the other ecosystem we manipulated community structure

122 by adding a competitor or a predator (see Fig. 1). We followed temporal changes in

123 community dynamics (i.e., species density and biomass) in each ecosystem, and then

124 compared the dynamics between connected and isolated ecosystems to test if

125 communities with different structure and composition could affect each other only

126 through subsidy exchanges. Specifically we asked whether autotroph versus

127 heterotroph community dynamics could have differential impacts on neighbours, and

128 whether the addition of a species inducing novel trophic interactions (e.g. competition or 129 predation) could affect neighbour dynamics in the absence of organism dispersal. 


\section{Methods}

\section{Experimental design}

To test the indirect influence of community composition on one another via cross-

134 ecosystem subsidies, we performed a two-patch meta-ecosystem experiment with

135 protists growing in microcosm ecosystems solely connected by subsidies (spatial flows

136 of detritus). We varied species traits in Ecosystem 1 and trophic structure in Ecosystem

1372 according to a factorial design (Fig. 1). In Ecosystem 1 we grew one of these three

138 species with contrasting traits alone (monocultures): the autotroph flagellate Euglena

139 gracilis ( $\sim 35 \mu \mathrm{m})$, a large bacterivorous ciliate (Paramecium aurelia, $\sim 96 \mu \mathrm{m})$, or a small

140 bacterivorous ciliate (Tetrahymena pyriformis, $\sim 30 \mu \mathrm{m}$ ). In Ecosystem 2, our focal

141 species, Colpidium striatum, a small bacterivorous ciliate $(\sim 4 \mu \mathrm{m})$ was grown alone, or

142 either with Paramecium aurelia as a competitor, or was submitted to predation by the

143 generalist filter feeding waterflea Daphnia magna ( 0.5 to $2 \mathrm{~mm})$. Treatments are

144 hereafter called "Autotroph", "Large heterotroph" and "Small heterotroph" in Ecosystem

1451 and "Focal Species" (FS), "FS+Competitor", "FS+Predator" in Ecosystem 2. We

146 connected Ecosystem 1 and Ecosystem 2 only by subsidy exchanges (detritus,

147 including inorganic resources present in the growing medium), and prohibited any type

148 of species dispersal. We had a five-fold replication of the nine meta-ecosystem types (all

149 possible combinations of Ecosystems 1 and 2), as well as the control ecosystems

150 without diffusion, resulting in an experiment consisting of 45 independent 2-patch meta-

151 ecosystems and 30 isolated 1-patch ecosystems (120 microcosms). 


\section{Experiment setup}

154 Ecosystems were assembled in microcosms of $250 \mathrm{~mL}$ Schott bottles filled with $100 \mathrm{~mL}$

155 culture medium. All organisms (bacteria, protists, and waterfleas) used in the experiment

156 were grown in pre-autoclaved protist pellet suspension filtered through Whatman filters

157 (0.31 g protist pellets $1 \mathrm{~L}^{-1}$ tap water; Protist pellets from Carolina Biological Supply,

158 Burlington NC, USA). Microcosms were assembled with $75 \mathrm{ml}$ protist medium, $5 \mathrm{~mL}$ of

159 bacterial culture (Serratia fonticola, Bacillus subtilis and Brevibacillus brevis; added one

160 day prior to protist addition), and completed with $20 \mathrm{~mL}$ of protist culture at carrying

161 capacity (10 $\mathrm{mL}$ per species in the competition treatment). For the predation treatment,

162 we added four equal-sized juvenile Daphnia magna to each microcosm. More details on

163 protist culture and experimental procedures are found in Altermatt et al. (2015).

164 Microcosms were randomized within the 5 replicate blocks, and the experiment took

165 place at $20^{\circ} \mathrm{C}$ and constant lighting. We replaced all Daphnia that died within the first

166 six days due to initial conditions (i.e., insufficient oxygen-levels in the freshly autoclaved 167 medium).

169 Diffusion

Ecosystem 1 and 2 were connected by bi-weekly reciprocal subsidy exchanges.

171 These spatial flow events consisted in sampling $30 \mathrm{~mL}$ from each ecosystem,

172 microwaving these samples for 100 seconds, until they reached a full boil, to turn all

173 living biomass into detritus, and then adding it into the respective connected ecosystem

174 after a cooling period of two hours at ambient temperature $\left(20^{\circ} \mathrm{C}\right)$. This diffusion 
175 method mimics detritus flows associated with recurrent perturbation-induced mortality.

176 Isolated controls were also submitted to the same perturbations, but the microwaved

177 samples were added back in the microcosm of origin to isolate the diffusion effects from

178 perturbation-induced mortality.

180 Samplings and measurements

181 In parallel, we tracked changes in community dynamics in each microcosm during

182 one month. We measured population densities every Tuesday and Friday (i.e., twice a

183 week), leaving as much time as possible for protist growth after each diffusion event

184 (occurring on Wednesdays and Saturdays). At each measurement point we took two 0.5

$185 \mathrm{~mL}$ samples per microcosm, one to measure protist density by video analysis, the other

186 to measure bacterial dynamics by flow cytometry. The volumes sampled were

187 considered sufficiently small to not affect local dynamics (Altermatt et al. 2011) and thus

188 did not need to be replaced by fresh medium. We recorded one $5 \mathrm{~s}$-video per sample

189 following a standardized video procedure (Pennekamp and Schtickzelle 2013, Altermatt

190 et al. 2015). From each video we extracted densities of moving individuals along with

191 morphometric and movement information, such as cell size and speed. This was

192 achieved using the R-package bemovi (Pennekamp et al. 2015) coupled to the image

193 analysis free-ware ImageJ (ImageJ, National Institute of Health, USA). The additional

194 traits measured served to discriminate species identity in mixtures (competition

195 treatment), provided as inputs to trait distance analysis with the svm algorithm (e1071 R-

196 package Meyer et al. 2014), and to calculate bio-areas as a proxy for biomass. Since

197 automated particle detection relies on movement, the method systematically 
198 underestimated densities of Euglena gracilis, which is much less mobile than the other

199 species. Therefore, we complemented density estimates of this species with visual

200 counts from the videos. To exclude false positives, we also performed a visual check of

201 all videos where a protist species had less than 3 individuals per frame. We counted

202 Daphnia individuals visually. Finally, we measured the total abundance of the 3-species

203 bacterial community on a flow cytometer (BD Accuri ${ }^{\mathrm{TM}} \mathrm{C} 6$ cell counter) on SYBR green

204 fixed samples (dilution x1000), following a standard protocol (Altermatt et al. 2015).

205

206 Analyses

207 We characterized the indirect effects of neighbouring communities on each other 208 through subsidy flows using log response-ratios of protist densities at each time point, 209 for the different neighbour treatments (either species traits or trophic structure) in the 210 connected ecosystem, with 95\% confidence intervals $(\mathrm{Cl})$. The responses were tested

211 relative to controls without diffusion, such that Cls not comprising zero reveal significant 212 effects of diffusion, while none overlapping Cls between neighbour treatments reveal a 213 significant neighbour effect. We tested the effects of trophic structure on neighbour 214 community dynamics by comparing the density of species in Ecosystem 1 (each 215 monoculture) when connected to different communities driven by specific interactions in 216 Ecosystem 2 (i.e. FS, FS+Competitor, FS+Predator). Conversely, we tested the effects 217 of functional traits on neighbour community dynamics by comparing the density of our 218 focal species, Colpidium striatum, in Ecosystem 2 when connected to different species 219 population with specific functional traits in Ecosystem 1 (i.e. Autotroph, Large 220 heterotroph, Small heterotroph). We studied the potential interaction with time by 
221 running generalized linear models (GLM) on log response-ratios with species traits or

222 trophic interaction in the connected ecosystem and time as explanatory variables. We 223 used Gaussian distributions as link functions to avoid overestimation of positive effects

224 due to the ratio (Berlow et al. 1999). The effect of species traits in Ecosystem 1 on

225 competitive outcome in Ecosystem 2 was also tested with a GLM on the density of

226 Colpidium relative to Paramecium (using the relative proportion), with species traits in

227 the connected ecosystem and time as explanatory variables. For each GLM, the level of

228 significance and the effect size of each factor, and their interactions, were assessed

229 using a standard F test (Type II analysis of deviance). For each significant term of

230 interest we ran post-hoc pairwise comparisons (with Tukey adjusted p-values) to

231 evaluate specific contrast among variables. When time was not significantly affecting the

232 terms of interest we performed the post-hoc analysis on simplified GLM sub-models

233 (without the effect of time) in order to avoid unnecessary inflations of time II error 234 (Nakagawa 2004).

Lastly, because neighbour effects can originate from changes in both subsidy 236 quantity and quality, we examined approximated biomass (bio-areas) at the different 237 trophic levels (bacteria, protist, predator) according to community characteristics 238 (species traits or trophic structure) to bring an element of interpretation, assuming that 239 the different trophic levels produce different qualities of detritus. For bacteria we took a 240 constant mean individual area of $1 \mu \mathrm{m}^{2}$, assuming no significant change of size over the 241 experiment (bio area $=1 \mu \mathrm{m}^{2}{ }^{*}$ cell counts). For protists, we measured mean individual's 242 area directly from the video analysis, and extrapolated it to the total population

243 (Pennekamp et al. 2015). Bio-area of Daphnia was estimated visually based on four size 
244 classes. To test protist and bacteria bio-area differences among communities along time 245 we ran four different GLM with either species traits (Ecosystem 1) or trophic structure 246 (Ecosystem 2) and time as explanatory variables. We studied residual distributions to 247 select the most appropriate link function (Gamma or Gaussian). All analyses were 248 conducted with R 3.1.2 (R Development Core team, 2014), using the 'car' package (Fox 249 and Weisberg 2011) for type II analysis of deviance, and the 'Ismeans' package for post 250 hoc pairwise comparisons (Lenth 2013). 


\section{Results}

252 Subsidy-mediated effects on population density

Both local community functional traits (autotrophy versus heterotrophy) and

254 trophic structure induced strong significant effects on connected communities via

255 subsidy exchanges and in the absence of dispersal (see Supplementary material

256 Appendix 1 Table A1 and Appendix 2 Table A2). At the end of the experiment,

257 population densities of our focal species in Ecosystem 2 were significantly higher when

258 connected to autotroph compared to heterotroph communities $\left(F_{2,60}=39.40, p<0.0001\right.$

259 in Focal Species and FS+Competitor treatments (see Supplementary material Appendix

2601 Table A1, and Appendix 5 Table A5 for post-hoc multiple comparisons) or compared

261 to isolated communities (Fig. 2, panels a and b). The only exception was in the

262 FS+Predator treatment where predation resulted in the extinction of the focal species

263 irrespective of functional traits in the connected community (Fig. 2c). Predators drove

264 protist to extinction and concentrated all the biomass in their body, which cascaded on

265 detritus quality and negatively affected population density in subsidy-connected

266 communities in Ecosystem 1 compared to the focal species grown alone (FS) (Fig. 3,

$267 p<0.0001$ for the factor "trophic structure" on each community; see Supplementary

268 material Appendix 1 Table A2 for the GLM and Appendix 5 Tables A5 for post-hoc

269 multiple comparisons). Moreover, the benefit (or not) of being a connected versus an

270 isolated community depended on species functional traits. Some species were relatively

271 insensitive to spatial subsidies when connected to FS or FS+Competitor communities

272 (e.g. Large heterotroph, Fig. 3b). In contrast, the autotroph did always far better when

273 connected to heterotroph communities, regardless of the trophic structure, compared to 
274 isolated autotroph communities, which received only their own autotroph detritus (Fig.

275 3a: confidence intervals above the zero line). At last, consumer size (Large versus Small 276 heterotroph) did not impact significantly species density in the connected ecosystem.

278 Subsidy-mediated effect on competition

While trophic structure in one ecosystem significantly impacted community

280 dynamics in the connected ecosystem, the reverse was also true: we found that the 281 competition outcome (in Ecosystem 2) strongly depended on species functional traits in

282 the connected ecosystem (Fig. 4, $F_{3,144}=8.56, p<0.0001$; see Supplementary material

283 Appendix 3 Tables A3 for full results of the GLM). At the end of the experiment, 284 coexistence between the two competitors (Colpidium and Paramecium) was only 285 observed in the meta-ecosystems with the autotroph as a neighbour (Fig. 4a). In the 286 presence of heterotrophic neighbours, we systematically observed competitive exclusion 287 of our focal species (Colpidium) by its competitor (Fig. 4b-d, with an exception in one 288 isolated replicate ecosystem), while it persisted over the course of the experiment when 289 connected to the autotroph community (Fig. 4a).

291 Changes in biomass distribution

We observed that the biomass distribution among trophic compartments (bacteria 293 / protists / predator) varies among community types, as inferred from bio-areas (Fig. 5; 294 Supplementary material Appendix 4 Tables A4). In the ecosystems where the predator 295 was present, both bacteria and protist biomass decreased dramatically within a few days 
296 (Fig. 5, right side), and almost all biomass was concentrated at the predator level 297 (99.97\% of the estimated bio-area at the end of the experiment). Protist and bacteria 298 biomasses did not differ between FS and FS+Competitor communities, but were 299 significantly lower in the FS+Predator community (adjusted p-value $<0.0001$ for every 300 pairwise comparison; see Supplementary material Appendix 5 Tables A5). Interestingly, 301 positive neighbour effects of the autotroph species on neighbour ecosystems occurred 302 despite the fact that autotroph protist biomass was generally lower or did not differ from 303 other neighbours (Fig. 5a, left side), but were associated with significantly higher 304 bacterial biomass (Fig. 5b, left side, see Supplementary material Appendix 5 Tables A5 305 for all multiple comparisons). 


\section{Discussion}

Community ecology usually assumes that species dispersal and local interaction

308 dynamics are the main processes driving regional biodiversity and species coexistence

309 (Leibold et al. 2004, Holyoak et al. 2005). With our experiment, we show that exchange

310 of subsidies among ecosystems also shapes community dynamics by mediating indirect

311 interactions between communities. By integrating resource dynamics in meta-

312 communities, the meta-ecosystem concept offers an operational framework to envision

313 spatial feedbacks between resource and community dynamics at a large spatial scale

314 (Loreau et al. 2003). Here, we demonstrate in experimental meta-ecosystems that the

315 integration of feedbacks between community dynamics and spatial subsidies is needed

316 to understand ecosystem dynamics, and are essential to forecast the spatial spread of

317 local perturbations.

Our experiment reveals three striking subsidy-mediated effects among

319 communities solely connected by detritus spatial flows. First, autotroph communities

320 positively affected densities in the connected communities, such that the persistence of

321 our focal species in the presence of a competitor was tributary to these autotroph

322 subsidies. Second, population densities in the autotroph community heavily depended

323 on subsidies from heterotroph communities. Third, by contrast to these positive effects,

324 local predation negatively affected connected communities, even though the predator

325 was not allowed to disperse, but was only indirectly affecting them through the alteration

326 of subsidy flows. These three effects demonstrate that local community composition and

327 structure can strongly affect community dynamics in neighbouring ecosystems, even in

328 the absence of dispersal. 
We explain the observed effects in terms of subsidy changes in quantity and

330 quality. The specific functioning of autotroph, heterotroph, and predation-pressured

331 communities impacted subsidies in contrasting ways. Photosynthetic activity by

332 autotrophs introduced new resources in the system otherwise closed, by fixing

333 atmospheric carbon, stored as carbohydrates (labile forms of carbon) or dissolved in the

334 medium by release activity (Baines and Pace 1991, Biersmith and Benner 1998, Guenet

335 et al. 2010). The greater bacteria densities in autotroph compared to heterotroph

336 communities likely profited from the exploitation of photosynthesis-derived dissolved

337 carbon (Børsheim et al. 2005). In addition, the small size of bacteria and their higher

338 surface/volume ratio may have enhanced recycling rates due to a greater proportion of

339 dead material exposed to decomposition (Berg 1984). Overall, even if not directly

340 measured, subsidies flowing from autotroph communities were undoubtedly more

341 abundant and more readily available than the ones flowing from heterotroph

342 communities. These rich subsidies subsequently triggered a bottom-up effect in the

343 neighbouring community resulting in higher densities of bacterivorous consumers.

344 Interestingly in the reverse direction also, subsidies from heterotroph communities

345 positively impacted autotroph community dynamics. This result stresses that neighbour

346 effects may not only happen via changes in subsidy abundance but also through

347 alterations to detritus stoichiometric balance. While not directly measured, the generally

348 greater ratio of nitrogen to carbon in heterotroph organisms (Elser et al. 2000, Sterner

3492009 ) is a straightforward explanation for the higher densities of autotroph communities

350 when exchanging detritus with heterotroph communities compared to isolated controls,

351 along with likely ammonium release activity of the bacterivorous protists (Probyn 1987). 
352 Overall, in our autotroph-heterotroph coupled ecosystems, heterotroph subsidies likely

353 relaxed autotroph nitrogen limitation, while autotroph subsidies fuelled heterotroph

354 growth with abundant labile carbon. Ecosystem couplings resulting in such resource

355 spatial complementarity are common between freshwater and riparian systems, with

356 riparian ecosystems exchanging abundant leaf litter against nitrogen-rich aquatic insects

357 (Baxter et al. 2005, Bartels et al. 2012), and thus highlight the general significance of our 358 findings.

By contrast, subsidies from communities with predators had negative effects on

360 neighbour community dynamics. We used a generalist predator species, capable of

361 consuming almost all protists and bacteria. Consequently, most of the biomass in these

362 ecosystems was concentrated at the highest trophic level, in the chitinous exoskeleton

363 of Daphnia. The molecular robustness of chitin as well as the low proportion of organic

364 material exposed to decomposition of larger organisms likely slowed down the recycling

365 process of predator detritus compared to other detritus (Berg 1984, Hamre et al. 2014).

366 The delay between slow recycling process and community dynamics triggered a spatial

367 cascade, leading to the accumulation of subsidies into recalcitrant form and to the

368 progressive starvation of neighbouring communities. It is noteworthy that in our closed

369 meta-ecosystems and in the absence of autotrophic species, the amount of subsidy

370 transferred is always the same ( $30 \%$ of total volume), therefore ruling out any local

371 collapse of subsidy to explain the negative effect of the predation. Therefore, the

372 observed indirect negative impact of predation on neighbour density likely comes from a

373 lower quality of subsidies. Beyond the specific effect of this particular predator, this

374 result stresses that changes in biomass distribution within a community may affect 
375 neighbours by modifying subsidy characteristics.

$376 \quad$ Overall, our experiment shows general mechanisms by which natural

377 communities can indirectly but strongly influence each other's functioning, via the

378 abundance, stoichiometry and decomposability of detritus locally produced and

379 subsidizing other ecosystems. Measuring directly the stoichiometric changes in

380 subsidies produced by contrasted communities could be a natural extension of this

381 experiment to investigate these mechanisms further. We demonstrate that, next to

382 species dispersal (for example tested by Staddon et al. (2010)), spatial dynamics of

383 detritus are essential by themselves to understand the fundamental functioning of

384 connected ecosystems, as well as their response to perturbations. The importance of

385 subsidies is often studied only from the recipient ecosystem's local perspective (see

386 Marcarelli et al. 2011, Sitters et al. 2015 for reviews), and ecosystem managers usually

387 consider the threat of direct alterations to resource flows (e.g. nutrient pollution), but

388 rarely threats potentially induced by alterations of community structure or composition in

389 connected ecosystems (but see the crash of forest spider communities, induced by

390 prey-subsidy disruption following the invasion of a stream by an exotic fish in Baxter et

391 al. 2004). Our results suggest that any kind of perturbation (e.g. land-use change, over-

392 harvesting) can have wider repercussions in space than those observed on local

393 communities because of subsidy disruption (e.g. cascades across aquatic-terrestrial

394 boundaries: Greig et al. 2012, Fey et al. 2015, Schulz et al. 2015). The extent to which

395 local perturbations spatially cascade to other communities will depend on the strength of 396 the subsidy coupling between ecosystems, an essential piece of information on which 397 more research is needed. Our findings call for the adoption of a spatial perspective in 
398 ecosystem management and restoration ecology that integrates fine-tuned knowledge of 399 resource spatial exchanges between ecosystems. 


\section{Acknowledgements}

401 We thank the EMERGe research group, Tom Battin and Anita Narwani for discussions,

402 Esther Keller and Elvira Mächler for technical support and Esther Keller and Piet Spaak

403 for providing the Daphnia magna.

404 


\section{Statement of authorship}

406 IG, EH and FA designed the research; IG, EH and PG conducted the research and

407 processed the data; IG analysed the data and wrote the first draft of the manuscript. All

408 authors contributed to the final version of the manuscript.

409 


\section{Funding}

411 This work was supported by the Swiss National Science Foundation Grant

412 PP00P3_150698, University of Zurich and Eawag.

413

414 


\section{References}

416

417 Allison, S. D. 2012. A trait-based approach for modelling microbial litter decomposition. $418 \quad$ Ecol. Lett. 15: 1058-70.

419 Altermatt, F., Schreiber, S. and Holyoak, M. 2011. Interactive effects of disturbance and 420 dispersal directionality on species richness and composition in metacommunities. $421 \quad$ Ecology 92: 859-870.

422 Altermatt, F. et al. 2015. Big answers from small worlds: A user's guide for protist 423 microcosms as a model system in ecology and evolution. - Methods Ecol. Evol. 6: $424 \quad 218-231$.

425 Baines, S. B. and Pace, M. L. 1991. The production of dissolved organic matter by 426 phytoplankton and its importance to bacteria: Patterns across marine and 427 freshwater systems. - Limnol. Oceanogr. 36: 1078-1090.

428 Bartels, P. et al. 2012. Reciprocal subsidies between freshwater and terrestrial 429 ecosystems structure consumer resource dynamics. - Ecology 93: 1173-1182.

430 Baxter, C. V. et al. 2004. Fish Invasion Restructures Stream and Forest Food Webs By. $431 \quad$ - Ecology 85: 2656-2663.

432 Baxter, C. V. et al. 2005. Tangled webs: Reciprocal flows of invertebrate prey link 433 streams and riparian zones. - Freshw. Biol. 50: 201-220.

434 Berg, B. 1984. Decomposition of root litter and some factors regulating the process:

435 Long-term root litter decomposition in a scots pine forest. - Soil Biol. Biochem. 16: 

$609-617$.

437 Berlow, E. L. et al. 1999. Quantifying variation in the strengths of species interactions. $438 \quad$ Ecology 80: 2206-2224.

439 Biersmith, A. and Benner, R. 1998. Carbohydrates in phytoplankton and freshly 440 produced dissolved organic matter. - Mar. Chem. 63: 131-144.

441 Børsheim, K. Y. et al. 2005. Photosynthetic algal production, accumulation and release 442 of phytoplankton storage carbohydrates and bacterial production in a gradient in 443 daily nutrient supply. - J. Plankton Res. 27: 743-755.

444 Carpenter, S. R. et al. 2001. Trophic cascades, nutrients, and lake productivity: Whole445 lake experiments. - Ecol. Monogr. 71: 163-186.

446 Cole, J. J. and Caraco, N. F. 2001. Carbon in catchments: Connecting terrestrial carbon $447 \quad$ losses with aquatic metabolism. - Mar. Freshw. Res. 52: 101-110.

448 Cole, J. J. et al. 2006. Differential support of lake food webs by three types of terrestrial $449 \quad$ organic carbon. - Ecol. Lett. 9: 558-568.

450 Cornwell, W. K. et al. 2008. Plant species traits are the predominant control on litter 451 decomposition rates within biomes worldwide. - Ecol. Lett. 11: 1065-1071.

452 Dreyer, J. et al. 2015. Quantifying aquatic insect deposition from lake to land. - Ecology 453 96: 499-509.

454 Elser, J. J. et al. 2000. Nutritional constraints in terrestrial and freshwater food webs. $455 \quad$ Nature 408: 578-580.

456 Enriquez, S. et al. 1993. Patterns in decomposition rates among photosynthetic 

organisms: the importance of detritus C:N:P content. - Oecologia 94: 457-471.

458 Fey, S. B. et al. 2015. Recognizing cross-ecosystem responses to changing temperatures: soil warming impacts pelagic food webs. - Oikos 124: 1473-81.

460 Fitzgerald, S. A. and Gardner, W. S. 1993. An algal carbon budget for pelagic-benthic $461 \quad$ coupling in Lake Michigan. - Limnol. Oceanogr. 38: 547-560.

462 Fox, J. and Weisberg, S. 2011. An $\{$ R $\}$ Companion to Applied Regression. - Sage.

463 Frank, K. T. et al. 2005. Trophic cascades in a formerly cod-dominated ecosystem. $464 \quad$ Science. 308: 1621-3.

465 García-Palacios, P. et al. 2015. The importance of litter traits and decomposers for litter 466 decomposition: a comparison of aquatic and terrestrial ecosystems within and $467 \quad$ across biomes. - Funct. Ecol. in press.

468 Gratton, C. and Vander Zanden, M. J. 2009. Flux of aquatic insect productivity to land: 469 Comparison of lentic and lotic ecosystems. - Ecology 90: 2689-2699.

470 Greig, H. S. et al. 2012. Warming, eutrophication, and predator loss amplify subsidies 471 between aquatic and terrestrial ecosystems. - Glob. Chang. Biol. 18: 504-514.

472 Guenet, B. et al. 2010. Priming effect: bridging the gap between terrestrial and aquatic 473 ecology. - Ecology 91: 2850-2861.

474 Hall, R. O. et al. 2000. Organic Matter Flow in Stream Food Webs With Reduced Detrital $475 \quad$ Resource Base. - Ecology 81: 3445-3463.

476 Hamre, A. G. et al. 2014. Enzyme processivity changes with the extent of recalcitrant 477 polysaccharide degradation. - FEBS Lett. 588: 4620-4624. 
478 Harvey, E., Gounand, I., Ganesanandamoorthy, P. and Altermatt, F. 2016. Spatially 479 cascading effect of perturbations in experimental meta-ecosystems. - Proceeding of 480 the Royal Society B-Biological Sciences 283: 20161496.

481 Hocking, M. D. and Reimchen, T. E. 2009. Salmon species, density and watershed size 482 predict magnitude of marine enrichment in riparian food webs. - Oikos 118: 1307483 1318.

Holyoak, M. et al. 2005. Metacommunities: spatial dynamics and ecological communities 485 (M Holyoak, MA Leibold, and RD Holt, Eds.). - University of Chicago Press.

486 Jackson, J. B. et al. 2001. Historical overfishing and the recent collapse of coastal $487 \quad$ ecosystems. - Science. 293: 629-37.

488 Leibold, M. A. et al. 2004. The metacommunity concept: a framework for multi-scale 489 community ecology. - Ecol. Lett. 7: 601-613.

490 Lenth, R. V. 2013. Ismeans: Least-squares means. R package version 1.06-05. in press.

491 Loreau, M. et al. 2003. Meta-ecosystems: a theoretical framework for a spatial 492 ecosystem ecology. - Ecol. Lett. 6: 673-679.

493 Marcarelli, A. M. et al. 2011. Quantity and quality: unifying food web and ecosystem 494 perspectives on the role of resource subsidies in freshwaters. - Ecology 92: 1215$495 \quad 1225$.

496 Massol, F. et al. 2011. Linking community and ecosystem dynamics through spatial 497 ecology. - Ecol. Lett. 14: 313-23.

498 Massol, F. et al. (in review) How life-history traits affect ecosystem properties: Effects of 499 dispersal in metaecosystems. - Oikos. 
500 Menninger, H. L. et al. 2008. Periodical cicada detritus impacts stream ecosystem 501 metabolism. - Ecosystems 11: 1306-1317.

502 Meyer, D. et al. 2014. e1071: Misc Functions of the Department of Statistics (e1071), TU 503 Wien. R package version 1.6-3. http://CRAN.R-project.org/package=e1071. in 504 press.

505 Mouquet, N. and Loreau, M. 2002. Coexistence in Metacommunities: The Regional 506 Similarity Hypothesis. - Am. Nat. 159: 420-426.

507 Nakagawa, S. 2004. A farewell to Bonferroni: The problems of low statistical power and 508 publication bias. - Behav. Ecol. 15: 1044-1045.

509 Pennekamp, F. and Schtickzelle, N. 2013. Implementing image analysis in laboratory510 based experimental systems for ecology and evolution: A hands-on guide. $511 \quad$ Methods Ecol. Evol. 4: 483-492.

512 Pennekamp, F. et al. 2015. BEMOVI, software for extracting behavior and morphology 513 from videos, illustrated with analyses of microbes. - Ecol. Evol. 5: 2584-2595.

514 Polis, G. A. et al. 1997. Toward an integration of landscape and food web ecology: the 515 dynamics of spatially subsidized food webs. - Annu. Rev. Ecol. Syst. 28: 289-316.

516 Polis, G. A. et al. 2004. Food Webs at the landscape level (GA Polis, ME Power, and $517 \quad$ GR Huxel, Eds.). - The University of Chicago press.

518 Probyn, T. A. 1987. Ammonium regeneration by microplankton in an upwelling 519 environment. - Mar. Ecol. Prog. Ser. 37: 53-64.

520 Richardson, J. S. et al. 2010. Resource subsidies across the land-freshwater interface 521 and responses in recipient communities. - River Res. Appl. 26: 55-66. 
522 Schindler, D. E. and Scheuerell, M. D. 2002. Habitat coupling in lake ecosystems. 523 Oikos 98: 177-189.

524 Schmitz, O. J. et al. 2000. Trophic Cascades in Terrestrial Systems: A Review of the 525 Effects of Carnivore Removals on Plants. - Am. Nat. 155: 141-153.

526 Schulz, R. et al. 2015. Review on environmental alterations propagating from aquatic to 527 terrestrial ecosystems. - Sci. Total Environ. 538: 246-261.

528 Scott, N. A. and Binkley, D. 1997. Foliage litter quality and annual net N mineralization:

529 comparison across North American forest sites. - Oecologia 111: 151:159.

530 Seymour, M., Fronhofer, E.A. \& Altermatt, F. 2015. Dendritic network structure and 531 dispersal affect temporal dynamics of diversity and species persistence. - Oikos $532 \quad 124: 908-916$.

533 Shurin, J. B. et al. 2006. All wet or dried up? Real differences between aquatic and 534 terrestrial food webs. - Proc. Biol. Sci. 273: 1-9.

535 Sitters, J. et al. 2015. Spatial stoichiometry: cross-ecosystem material flows and their 536 impact on recipient ecosystems and organisms. - Oikos 124: 920-930.

537 Staddon, P. et al. 2010. Connectivity, non-random extinction and ecosystem function in 538 experimental metacommunities. - Ecol. Lett. 13: 543-552.

539 Sterner, R. W. 2009. Nutrient Stoichiometry in Aquatic Ecosystems. - In: Encyclopedia of $540 \quad$ Inland waters. pp. 820-831.

541 Sterner, R. W. and Elser, J. J. 2002. Ecological Stoichiometry. The Biology of Elements 542 from Molecules to the Biosphere. - Princeton University Press. 


\section{3}


544 Supplementary material (available online as Appendix oik-XXXXX at

$545<$ <ww.oikosjournal.org/readers/appendix>). Appendix 1-5

546 Data available from the Dryad Digital Repository: XX

547

548 


\section{Figure legends}

550

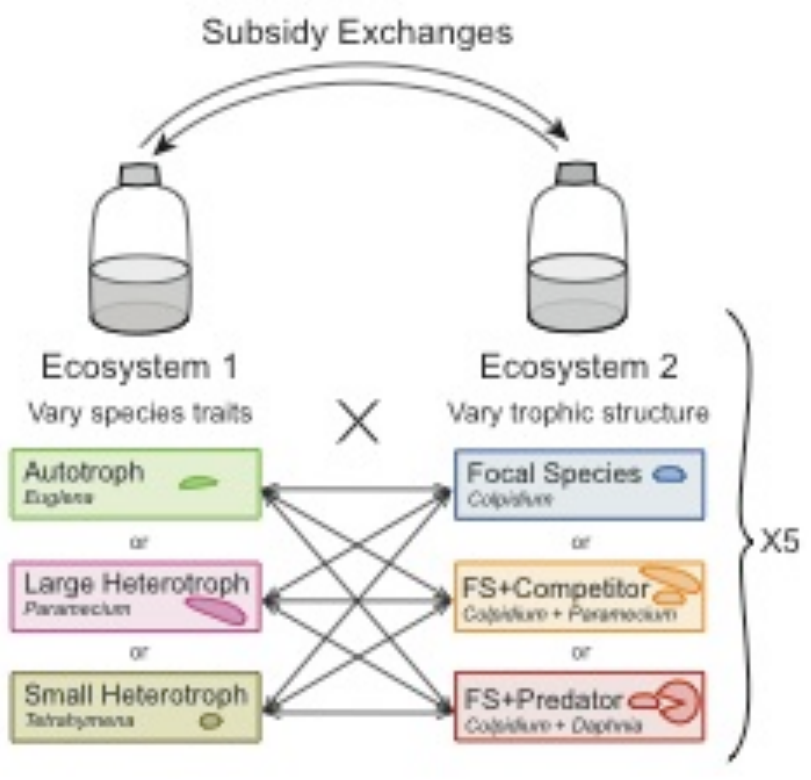

551 Figure 1. The experimental design consists of two-patch meta-ecosystems linked by

552 reciprocal exchanges of detritus and resources (subsidies). In Ecosystem 1, we varied

553 the dominant functional trait of the species present (either Euglena gracilis, Paramecium

554 aurelia, or Tetrahymena pyriformis). In Ecosystem 2, we varied the trophic structure, by

555 growing either a single species (Colpidium striatum; Focal Species (FS)), this same

556 species with an additional competitor (FS + Competitor: Colpidium striatum with

557 Paramecium aurelia), or in presence of a predator (FS + Predator: Colpidium striatum

558 with Daphnia magna). The combination of these two variation levels gives nine different

559 meta-ecosystems, each replicated five times. All ecosystems were inoculated with the

560 respective above named species, a bacterial community and organic resources. 


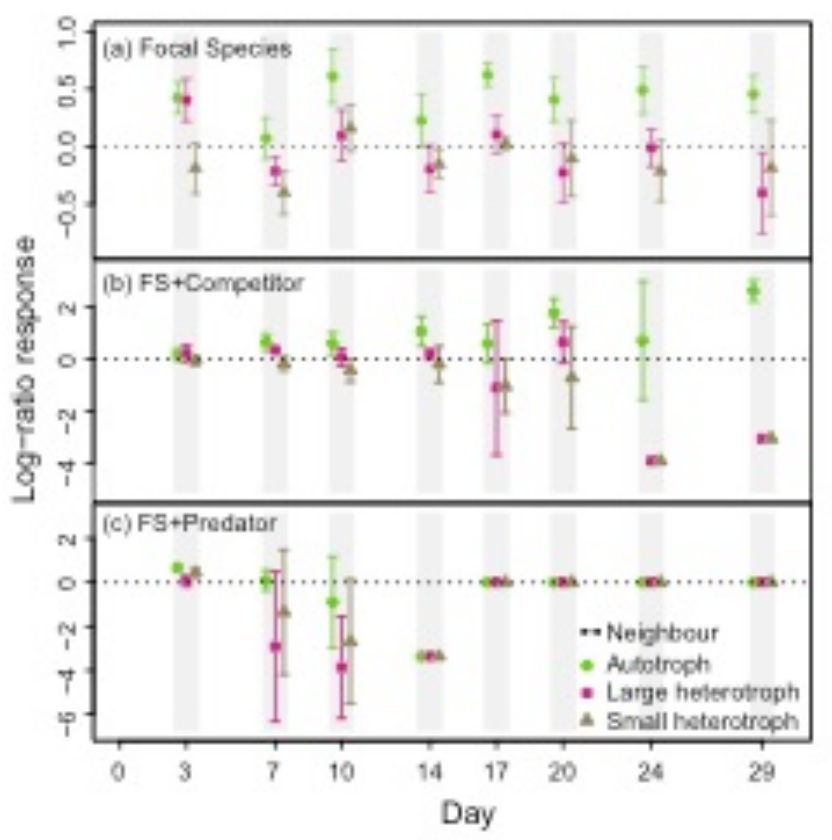

561

562 Figure 2. Effect of the species traits in Ecosystem 1 on the density of focal species in

563 Ecosystem 2 (Colpidium striatum) over time, expressed as the log response-ratio (RR)

564 of Colpidium density to detritus diffusion compared to the control without diffusion: Log

$565 \mathrm{RR}=\ln \left(\left(\mathrm{N}_{\mathrm{Col}}^{\text {diff }+}+\min _{\mathrm{D}}\right) /\left(\mathrm{N}_{\mathrm{Col}}^{\text {diff }-}+\min _{\mathrm{D}}\right)\right)$, with $\mathrm{N}$ the density and $\min _{\mathrm{D}}$ the minimal

566 density detectable by our video analysis. Shapes and colours refer to the neighbour

567 species. Bars give the $95 \%$ confidence interval $(\mathrm{Cl})$. Densities with $\mathrm{Cl}$ not crossing the

568 zero dotted lines differ significantly from the control. None overlapping Cls reveal protist

569 densities significantly differing from each other. Each panel gives the results for a

570 different trophic structure involving our focal species: (a) Focal Species, (b)

571 FS+Competitor, (c) FS+Predator. 


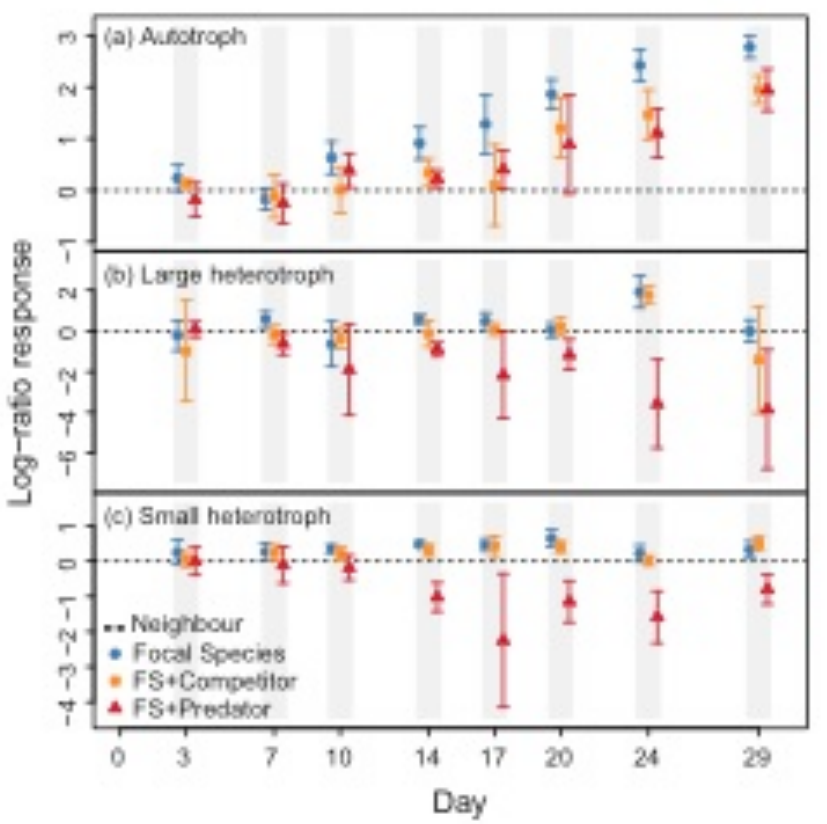

572

573 Figure 3. Effect of trophic structure in Ecosystem 2 on the density of different species in

574 Ecosystem 1 via the diffusion of detritus over time, expressed as the log-ratio of density

575 response to detritus diffusion compared to the control without diffusion: log response-

576 ratio $=\ln \left(\left(\mathrm{N}^{\text {diff }+}+\min _{\mathrm{D}}\right) /\left(\mathrm{N}^{\text {diff }-}+\min _{\mathrm{D}}\right)\right)$, with $\mathrm{N}$ the density and $\min _{\mathrm{D}}$ the minimal

577 density detectable by our video analysis. Shapes and colours refer to the neighbour

578 trophic structure. Bars give the 95\% confidence interval $(\mathrm{Cl})$. Densities with $\mathrm{Cl}$ not

579 crossing the zero dotted lines differ significantly from the control. None overlapping Cls

580 reveal protist densities significantly differing from each other. Each panel gives the

581 results for a different species with specific traits in Ecosystem 1: (a) Autotroph (Euglena

582 gracilis), (b) Large heterotroph (Paramecium aurelia), (c) Small heterotroph

583 (Tetrahymena pyriformis). 


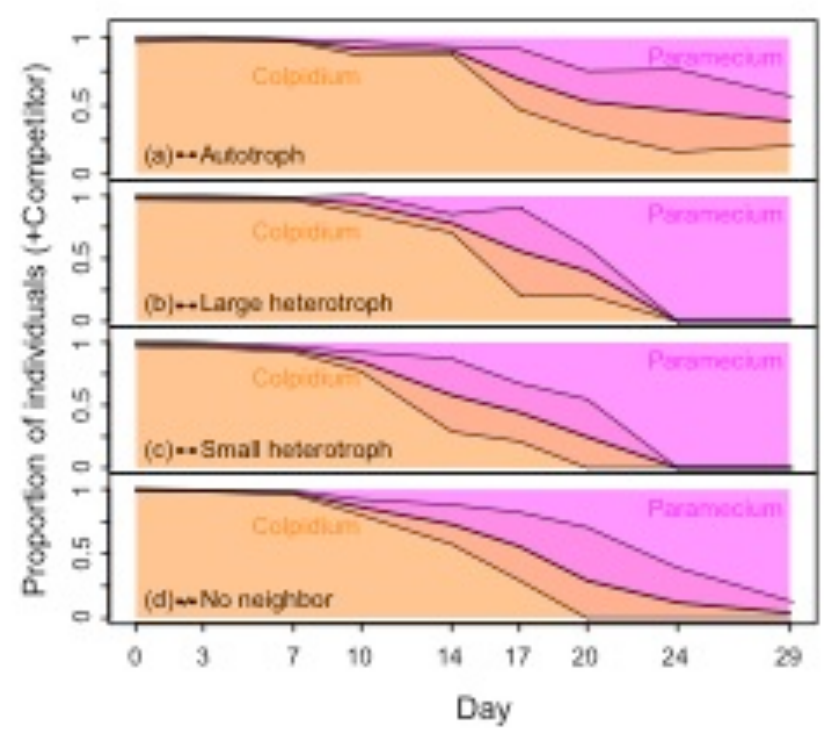

584

585 Figure 4. Effect of species trait in Ecosystem 1 on the competition hierarchy between

586 Colpidium (orange area) and Paramecium (pink area) in Ecosystem 2 over time

587 (FS+Competitor treatment). The effect is expressed as the relative proportion of

588 Colpidium versus Paramecium individuals (the two competitors). Neighbour communities

589 are either the Autotroph (Euglena gracilis), or the Large heterotroph (Paramecium

590 aurelia) or the Small heterotroph (Tetrahymena pyriformis), in panel (a), (b), and (c)

591 respectively. Barbells refer to subsidy connection. Panel (d) shows the control without

592 diffusion. Dotted lines give the standard deviation.

593 
594

595

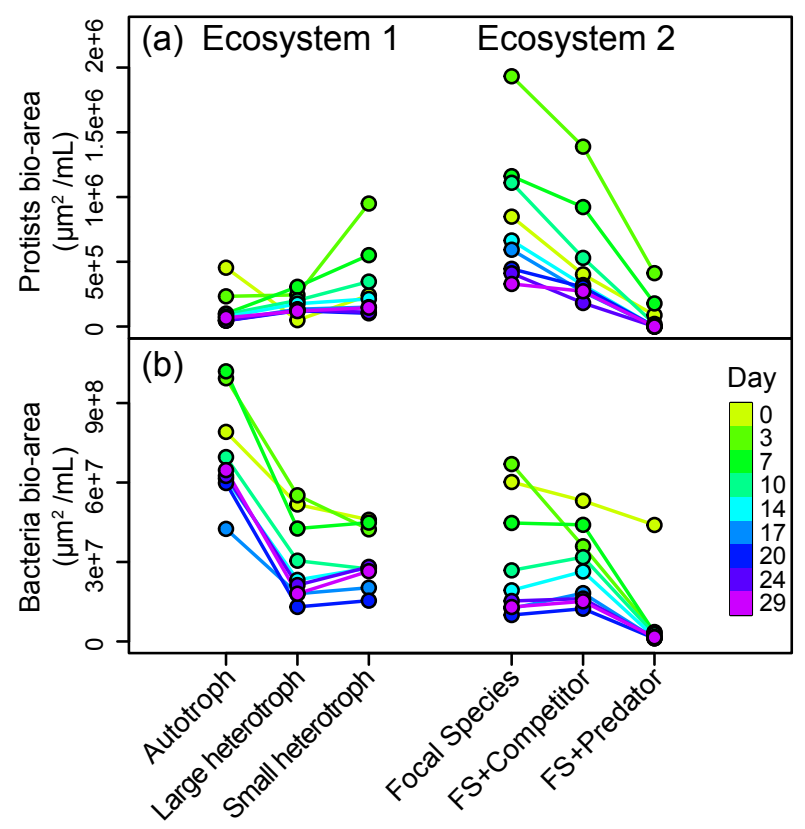

Community

596 Figure 5. Bio-area of (a) protists and (b) bacteria over time (colours) for the different

597 communities in each ecosystem. Points give the mean averaged over five replicates at a 598 specific day (colours). 